\title{
High sensation seeking is associated with behavioral and neural insensitivity to increased negative outcomes during decision-making under uncertainty
}

\author{
Sihua $\mathrm{Xu}^{1} \cdot$ Lijuan Luo $^{1} \cdot$ Zhiguo Xiao $^{2} \cdot \mathrm{Ke} \mathrm{Zhao}^{3} \cdot$ Hua Wang ${ }^{1,4} \cdot$ Cencen Wang ${ }^{1} \cdot$ Hengyi Rao $^{1,3}$
}

Published online: 28 October 2019

(C) The Psychonomic Society, Inc. 2019

\begin{abstract}
High-level sensation seeking (HSS) has been linked to a range of risky and unhealthy behavior; however, the neural mechanisms underlying such linkage remain unclear. In the present study, we used event-related potential (ERP) with a Balloon Analogue Risk Task (BART) to investigate how sensation seeking modulates brain responses to sequential decision-making with variable reward/loss outcome magnitudes. Behavior data showed that decision-making behavior was significantly affected by the large compared with the small magnitude of monetary outcome in the BART for individuals with low-level sensation seeking (LSS), but not for individuals with HSS. Similarly, HSS individuals displayed no changes in feedback-related negativity (FRN) in response to negative outcomes from decision-making with large or small reward/loss magnitudes, whereas LSS individuals showed greater FRN in response to decision-making with large loss magnitude than those with small loss magnitude. In addition, FRN amplitude differences correlated with decision-making behavior changes from small to large outcome magnitude for LSS, while such correlation was not observed for HSS. These findings suggest that a high-level of sensation seeking is associated with behavioral and neural insensitivity to increased negative outcome during decision-making under uncertainty, which may lead to greater risky behavior in these individuals when facing potential loss.
\end{abstract}

Keywords Sensation seeking · Feedback-related negativity · Balloon Analogue Risk Task

Human decision-making behaviors are influenced by a number of personality factors, including sensation seeking and impulsivity. Sensation seeking, a personality trait defined as the seeking of varied, novel, complex, and intense sensations and experiences, and willingness to take physical, social, legal, and financials risks for the sake of such experience (Zuckerman, 1994), has been linked to participation in various unhealthy and risky activities, including risky driving (Jonah,

Electronic supplementary material The online version of this article (https://doi.org/10.3758/s13415-019-00751-x) contains supplementary material, which is available to authorized users.

Sihua $\mathrm{Xu}$

sihuaxu@shisu.edu.cn

Lijuan Luo

luolijuan100@126.com

Hengyi Rao

hengyi@pennmedicine.upenn.edu

1 Laboratory of Applied Brain and Cognitive Sciences, School of Business and Management, Shanghai International Studies University, 550 Dalian West Street, Shanghai 200083, China
1997), excessive gambling (Harris, Newby, \& Klein, 2015), physically risky sports (Ruedl et al., 2015), aggressive and/or unsocialized behavior (Wilson \& Scarpa, 2011), and substance use (Bardo et al., 2007). Compared to individuals with the low sensation-seeking (LSS) trait, individuals with the high sensation-seeking (HSS) trait tend to exhibit greater subjective rewarding effects when using psychostimulants, such as alcohol and d-amphetamine, or when ignoring traffic rules 
(Doran et al., 2011; Fillmore, Ostling, Martin, \& Kelly, 2009; Iversen \& Rundmo, 2002; Lang et al., 2012; Weiland et al., 2013). HSS individuals also show more blunted monetary losses (Kruschwitz, Simmons, Flagan, \& Paulus, 2012), suggesting that HSS individuals may be more likely to repeat and engage in activities that encompass increased risk or negative consequences (Horvath \& Zuckerman, 1993).

One potential explanation for the observed differences between HSS and LSS is that individuals with the HSS trait may have different arousal levels (Hebb, 1955) and/or resting heart rate (Schmidt, Mussel, \& Hewig, 2013) compared with individuals with the LSS trait. Specifically, HSS individuals may be unable to fully reach their optimal arousal levels and resting heart rates through daily activities; therefore, they may need to seek more exciting and novel experiences to achieve that. However, HSS individuals may not recognize or be aware of the potential adverse consequences of such exciting and novel experiences (Zuckerman \& Kuhlman, 2000).

Another potential explanation theory highlights the role of motivation in sensation seeking and argues that sensationseeking behavior may be mainly driven by a "hyperactive approach system" (Joseph, Liu, Jiang, Lynam, \& Kelly, 2009; Kruschwitz et al., 2012) and a "hypoactive avoidance system" (Zheng, Sheng, Xu, \& Zhang, 2014). According to this theory, HSS individuals tend to be hypersensitive to reward but hyposensitive to punishment compared with LSS individuals. Several previous studies have demonstrated that individuals with high sensation seeking and impulsivity levels did not reduce their risky decisions in the face of potentially large rewards/loss outcomes during dynamic decision-making tasks (Bornovalova et al., 2009; Xu, Fang, \& Rao, 2013). For example, Bornovalova et al. (2009) found that, as the rewards/ losses increased, there was a drop in uncertainty-bearing behavior for the LSS individuals, whereas HSS individuals were largely insensitive to variations in rewards/losses magnitude. Taken together, these findings provide consistent evidence supporting the linkage between different levels of sensation seeking and altered decision-making behavior and processing of reward/loss outcomes under uncertainty.

However, the neural mechanisms underlying this linkage remain uncertain. Several neuroimaging studies have attempted to uncover these neural mechanisms and found that HSS individuals exhibit greater brain activation for rewards/ gains (Kruschwitz et al., 2012), but reduced activation for errors, losses, and reward absences (Kruschwitz et al., 2012; Cservenka, Herting, Seghete, Hudson, \& Nagel, 2013). Recently, a functional magnetic resonance imaging (fMRI) study examined the decision-making process and found that HSS individuals relative to LSS individuals exhibited significantly reduced neural responses during risk-taking in multiple brain regions, including the thalamus and the dorsomedial prefrontal cortex (Zheng et al., 2017). While fMRI provides excellent spatial resolution for identifying brain regions, underlying decision-making behavior, and processing of rewards/loss outcomes, it lacks the high temporal resolution that is needed to precisely characterize the time course of activity therein. On the other hand, event-related potential (ERP) provides a more precise assessment of the time courses of neural activation during the implementation of risky or uncertain behaviors, which permit the separation of neural events occurring very closely in time (Luck, 2014).

The feedback-related negativity (FRN) is a specific ERP component frequently reported in many decision studies. This component denotes a negative deflection in the frontocentral sites and reaches its peak at approximately 200-300 ms after the onset of negative feedback (Miltner, Braun, \& Coles, 1997). FRN is considered as an index of outcome appraisal during decision-making and has been the focus of numerous studies (Fein \& Chang, 2008; Gehring \& Willoughby, 2002; Holroyd \& Coles, 2002; Xu et al., 2016, 2018). The FRN appears to originate from the anterior cingulate cortex (Gehring \& Willoughby, 2002) and is considered to reflect prediction errors of the actual outcome (Holroyd, Hajcak, \& Larsen, 2006). Variations in the FRN amplitude might be reflective of individual differences in the processing of assessing the motivational/affective significance of outcomes, that is, the processing of assigning subjective values or responsibility for the actions onto outcomes (Gehring \& Willoughby, 2002; Zhou, Yu, \& Zhou, 2010). The FRN also may be reflective of a fast motivational or affective reaction to the detection of the reward prediction errors (Massar, Rossi, Schutter, \& Kenemans, 2012). Additionally, a growing body of research has suggested that FRN is sensitive to variations in reward magnitude when using real money as the incentive (Bellebaum, Polezzi, \& Daum, 2010; Gu et al., 2011; Luo \& Qu, 2013; Wu \& Zhou, 2009; Xu et al., 2018). For example, our previous ERP study demonstrated that the amplitude of FRN in response to negative feedback during decisionmaking varies with large or small real monetary rewards $(\mathrm{Xu}$ et al., 2018).

Several previous studies have examined the relationships between the amplitude of FRN and sensation seeking personality traits but obtained mixed results (Leicht et al., 2013; Zheng \& Liu, 2015; Gu et al., 2017; Novak, Novak, Lynam, $\&$ Foti, 2016). For example, Leicht et al. (2013) used a simple two-choice gambling task and observed no direct correlations between the FRN amplitudes and sensation seeking scores. However, this study reported significant positive correlations between the alpha and low-beta power increases upon loss and the respective subscale scores of Sensation Seeking Scale. Zheng and Liu (2015) used a similar two-choice gambling task and reported that the FRN difference between loss and gain outcomes was more enhanced in the high-risk than in the low-risk conditions in LSS individuals but showed no differences in HSS individuals. Moreover, Novak et al. (2016) reported positive correlations between the FRN amplitude and 
sensation seeking scores in a monetary incentive delay task. However, these correlations failed to be replicated in a more recent ERP study using a similar monetary incentive delay task (Gu et al., 2017), which reported that the FRN amplitude varied as a function of disinhibition.

It is worth noting that many previous FRN studies used simple two-choice gambling tasks. However, the simple choices in these tasks measure only one dimension of decision-making (Helfinstein et al., 2014). From an economics perspective, the risk is typically defined as the degree of outcome variability, while risk-taking and uncertainty-bearing behavior is more likely a dynamic sequential process, involving a series of choices before the final positive or negative outcome. Dynamic sequential tasks are also more reflective of real-life decision-making situations than the simple twochoice gambling tasks. Neuroimaging studies have suggested different brain activation patterns between dynamic sequential decision-making tasks and two-choice simple gambling tasks (Boorman, Behrens, Woolrich, \& Rushworth, 2009; Schonberg et al., 2012). For example, ventromedial prefrontal cortex activity was shown to increase with the added value of an option in a two-choice simple gambling task (Boorman, Behrens, Woolrich, \& Rushworth, 2009) but was reported to decrease as accumulated values increased in the uncertaintybearing, decision-making task (Schonberg et al., 2012). However, it remains unclear whether HSS individuals would exhibit diminished sensitivity to variations in the risk levels and/or outcome magnitudes during dynamic sequential decision-making situations.

The Balloon Analogue Risk Task (BART) is a paradigm involving dynamic sequential decision-making under uncertainty (Schonberg et al., 2012). In this computerized task, participants are requested to press a button to sequentially pump air into a virtual balloon. With each button press to pump the balloon, participants see an increase in the amount of earned monetary reward. The task requires participants to balance between the desire for larger gains and the desire to avoid the uncertainty of reward forfeiture. Participants are given the opportunity to collect the reward or leave the risky situation at any time, as is often the case in real life decisionmaking situations (e.g., "Should I drink more?"). This characteristic of the BART gives it high external validity (Helfinstein et al., 2014; Lejuez et al., 2002).

The average number of pumps in the BART has been shown to correlate with self-reported risk-taking behaviors (Banducci et al., 2015). Previous studies have suggested that decision-making behavior on the BART was modulated by sensation seeking levels (Mishra, Lalumiere, \& Williams, 2010; Bornovalova et al., 2009; Xu et al., 2013). Specifically, uncertainty-bearing behavior for the LSS individuals decreased as reward/loss magnitude increased, whereas those for the HSS individuals were largely insensitive to variations in reward/loss magnitude (Bornovalova et al., 2009;
Xu et al., 2013). A growing number of ERP studies have used the BART to evaluate the neural activity patterns during dynamic sequential decision-making (Euser, Van Meel, Snelleman, \& Franken, 2011; Fein \& Chang, 2008; Kóbor et al., 2015; Kessler, Hewig, Weichold, Silbereisen, \& Miltner, 2017; Kiat, Straley, \& Cheadle, 2015). The changes of FRN amplitudes have been shown to correlate with risktaking behavior changes from small to large real monetary rewards but not with risk-taking behavior changes from small to large hypothetical monetary rewards, suggesting that real and hypothetical money incentives may have different effects on risk-taking behavior and brain activity (Xu et al., 2018). Currently, there remains a paucity of studies examining the neural basis underlying potential different sequential decision-making behavior in HSS versus LSS individuals. To address this question, we used ERP in this study and examined both behavior and brain activity changes in high and low sensation seekers during performing the BART with large or small reward/loss magnitudes. We hypothesized that HSS individuals would behave more impulsively in face of large potential reward/loss magnitude and show diminished changes in the FRN amplitude in response to varied reward/loss magnitudes in the BART compared with LSS individuals.

\section{Methods}

\section{Participants}

Forty-two undergraduate students $(n=21$ females, mean age $19.88 \pm 1.42$ years) voluntarily participated in the experiment. These participants were initially selected from a large university student sample $(N=360,202$ females and 158 males; Table 1) based on their scores on the Chinese version of Sensation Seeking Scale Form V (SSS-V; Wang et al., 2000; Zuckerman, 1994). The SSS-V comprises four 10 -item subscales: disinhibition, thrill and adventure seeking, boredom susceptibility, and experience seeking. Summing all 40 items yields an overall sensation-seeking score. We selected participants who scored in the top $20 \%$ of the score distribution as the HSS group and participants who scored in the bottom $20 \%$ as the LSS group. The criterion of selection was employed separately for males and females because of the gender imbalance in the sample. Potential participants were then invited randomly. The groups differed significantly on the overall sensation-seeking scores and its subscale scores and showed no differences in age, gender, and educational level (Table 1). All participants were right-handed, with normal or correctedto-normal vision, and had no history of neurological or psychiatric disorders or head trauma. We excluded participants who reported having drunk alcohol or smoked tobacco within two weeks before the test. Participants received a small payment of 30 Chinese yuan (approximately US $\$ 4.50$ ) for their 
Table 1 Sample characteristics $(\mathrm{M} \pm \mathrm{SD})$

\begin{tabular}{lllll}
\hline & Total $(\mathrm{n}=360)$ & High sensation seekers $(\mathrm{n}=21)$ & Low sensation seekers $(\mathrm{n}=21)$ & $p$ value \\
\hline Gender (M/F) & $158 / 202$ & $10 / 11$ & $11 / 10$ & 0.715 \\
Age (yr) & $19.86 \pm 0.56$ & $20.05 \pm 1.47$ & $19.71 \pm 1.38$ & 0.548 \\
Education (yr) & $13.76 \pm 1.46$ & $13.90 \pm 1.30$ & $13.67 \pm 1.28$ & 0.637 \\
Thrill and adventure seeking score & $6.25 \pm 0.98$ & $8.24 \pm 1.06$ & $4.29 \pm 1.48$ & $2.24 \pm 1.23$ \\
Experience seeking score & $4.64 \pm 1.89$ & $7.01 \pm 1.23$ & $1.90 \pm 1.15$ & $<0.001$ \\
Disinhibition scores & $3.87 \pm 1.64$ & $5.81 \pm 1.40$ & $1.29 \pm 0.82$ & $<0.001$ \\
Boredom susceptibility score & $3.08 \pm 1.34$ & $4.86 \pm 1.17$ & $9.71 \pm 2.35$ & $<0.001$ \\
Sensation seeking score & $17.6 \pm 4.86$ & $25.90 \pm 2.56$ & $<0.001$ \\
\hline
\end{tabular}

participation and a small bonus (up to 70 yuan, about $\$ 10.50$ ) based on their BART performance. This study protocol was reviewed and approved by the institutional review board of the Guangdong University of Finance and Economics. All participants provided written informed consent before their enrollment, as dictated by the Declaration of Helsinki.

\section{Task and procedure}

Participants sat approximately $70 \mathrm{~cm}$ from a computer screen in an electrically shielded room and completed the BART twice (Fig. 1). The BART was implemented with E-prime 1.1 (Psychology Software Tools, Inc., Pittsburgh, PA). They completed the task once during a low reward/loss magnitude condition ( 0.01 yuan/button press) and once during a high reward/loss magnitude condition ( 0.5 yuan/button press). The ordering of these conditions was randomized between subjects, and each condition could be completed in approximately $22 \mathrm{~min}$. Participants were told that they would be paid by the real monetary gains they earned in the task, because recent studies suggest that payment methods may have significant impacts on participants' choices and ERP amplitudes (Schmidt \& Hewig, 2015; Schmidt et al., 2019).

In each condition, there were 100 balloons to fill. Balloon stimuli were blue spheres with radii that increased proportionally to button press pumps (and the amount of money added to the temporary bank). The visual angle of the balloon before inflating was $4.5^{\circ}$, which increased by $0.3^{\circ}-0.54^{\circ}$, depending on the amount of money added to the balloon. The maximum breaking point for each balloon in the BART was 12 pumps. Thus, the probability of the balloon bursting after the first pump was $9.1 \%(1 / 11)$, after the second pump was $10 \%$ $(1 / 10)$, etc., until the 12 th pump, where the probability of the balloon popping was $100 \%(1 / 1)$. When a balloon was pumped past its individual popping point, all money earned for that balloon (which appeared in a temporary bank) was lost and the same amount of money was deducted from their total earnings. We removed the popping sound during the BART, because it might have induced unwanted startle responses or muscle artifact in the electroencephalographic (EEG) recordings for ERP measurement.

A trial ended when participants either chose to end the trial and collect all current earnings for that balloon (which they did by pressing a button) or the balloon was pumped past its individual popping point, at which point participants lost all previous earnings from that balloon and the same amount of earning was subtracted from their cumulative earnings as a penalty. At the end of each trial, a number (the amount of accumulated money for the current balloon) appeared at the center of the screen as feedback. We used red or green font color in the current study, because the monitoring system is sensitive to the most salient aspect of feedback information indicating monetary gains or losses (Nieuwenhuis et al., 2004), and it is easier for participants to extract gains or losses information from the feedback display, which is similar to Gehring and Willoughby's study (2002). For half of the subjects, red indicated monetary gains and green indicated monetary losses, and for the other half, the reverse was true. The delay between participants' response and the appearance of the feedback was randomized from 1.0 to $1.2 \mathrm{sec}$. Before conducting the formal experiment, participants were given the opportunity to practice the task until they orally reported that they had mastered it.

\section{Electroencephalogrphy recording and analysis}

Electroencephalogrphy (EEGs) were recorded while participants performed the BART (bandpass 0.1-100 Hz, sampling rate $512 \mathrm{~Hz}$ ) with the NeuroScan Synamps 2 EEG system (Charlotte, NC). Participants were outfitted with an elastic electrode cap with $64 \mathrm{Ag} / \mathrm{AgCL}$ electrodes arranged according to the International 10-20 system. EEG recordings were made using a common average reference online and then algebraically re-referenced to the average of the left and right mastoids of each participant. Electrode impedance was reduced to less than $3 \mathrm{k} \Omega$. In order to detect blinks and eye movements, horizontal and vertical electrooculograms were recorded. All the data were processed offline using MATLAB R2011b (Math Works, Natick, MA) and the 


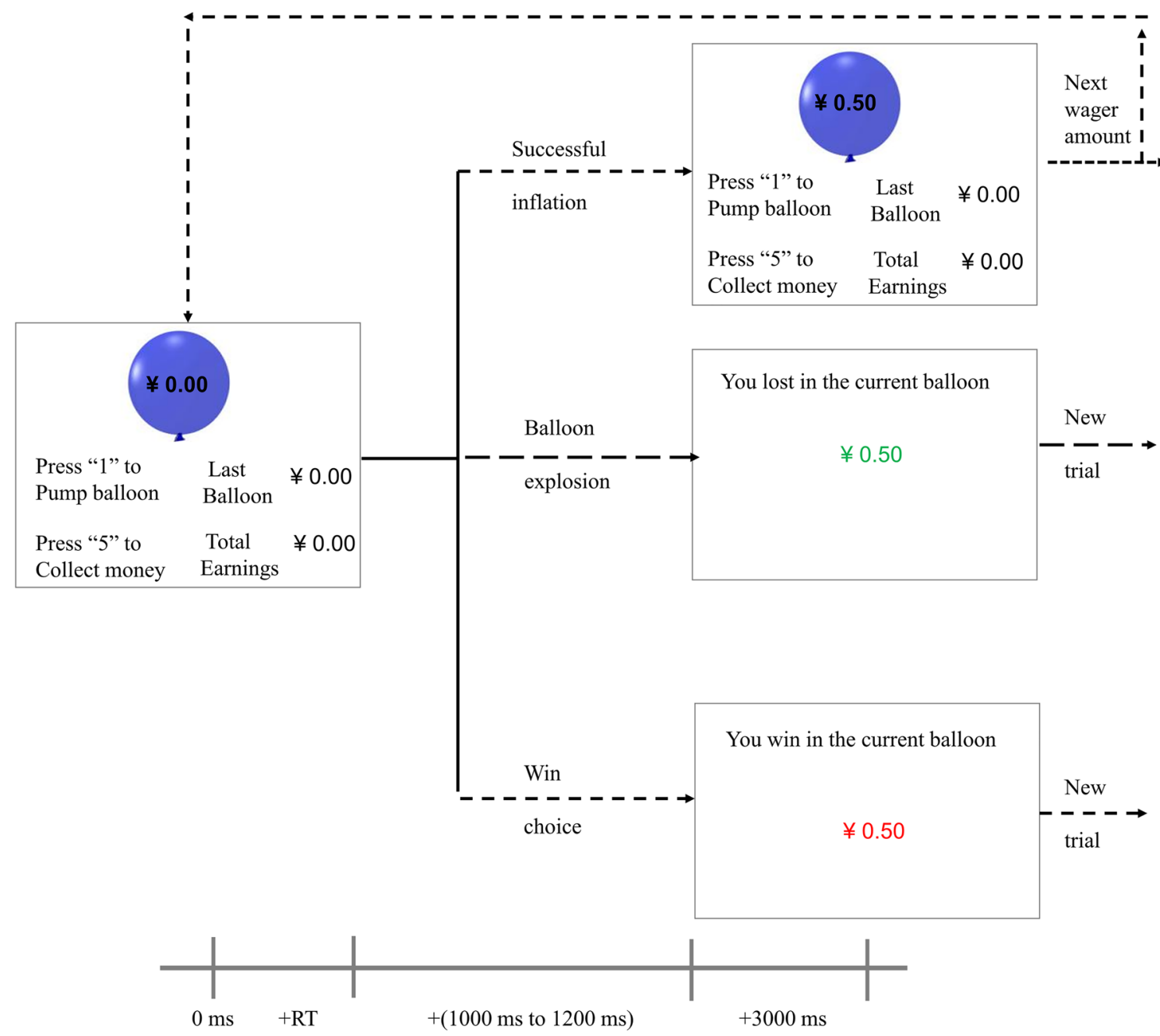

Fig. 1 Schematic diagram for the BART

Toolbox EEGLAB 12.0.1 (Delorme \& Makeig, 2004). The data were filtered using a $30-\mathrm{Hz}$ low-pass filter and a $0.1-\mathrm{Hz}$ high-pass filter. Each EEG epoch began $100 \mathrm{~ms}$ before stimulus onsets and ended 1,000 ms after onset. Before averaging, all data sets were corrected for eye-blink and movement artifacts by applying an Independent Components Analysis. Any trials contaminated by eye movements, eye blinks, or muscle potentials exceeding $\pm 100 \mu \mathrm{v}$ at any electrode were excluded. The baseline for ERP measurements was the average voltage recorded over the $100 \mathrm{~ms}$ prestimulus interval.

FRN was measured as the mean wave amplitudes over a time window of 200-280 ms after positive or negative feedback onset. Based on the topographical distribution of each ERP component (Fig. 2), and findings from previous studies (Euser et al., 2011; Fein \& Chang, 2008; Kóbor et al., 2015; Wu \& Zhou, 2009; Yeung \& Sanfey, 2004), the FRN was preliminarily calculated across three electrodes: $\mathrm{Fz}, \mathrm{FCz}$, and $\mathrm{Cz}$. We found that the FRN was strongest at the $\mathrm{Cz}$ site. Hence, we focused on the $\mathrm{Cz}$ electrode for more detailed analyses. Table 2 shows the number of trials in each condition. We observe no significant differences in trial counts between HSS and LSS individuals $(p>0.050)$. These numbers meet the recommendations by Marco-Pallares et al. (2011) for at least 20 trials to measure a stable FRN.

\section{Statistical analysis}

Descriptive data are presented as the mean \pm standard error below. The significance level was set at 0.05 . The statistical analysis was conducted with independent samples $t$ tests and mixed analyses of variance (ANOVAs) in SPSS 16.0 (SPSS Inc., 2007). Differences in gender, age, and education level were examined using independent-samples $t$ tests. Decisionmaking behavior and FRN were assessed with mixed ANOVAs. To control for Type I error, we applied Greenhouse-Geisser corrections with post hoc Bonferroni at a significance level of $p<0.050$. We also applied Spearman correlation coefficients to examine the associations between changes in FRN (? FRN) and changes in uncertainty-bearing behavior (i.e., average number of pumps; ?decision-making 
High sensation seekers
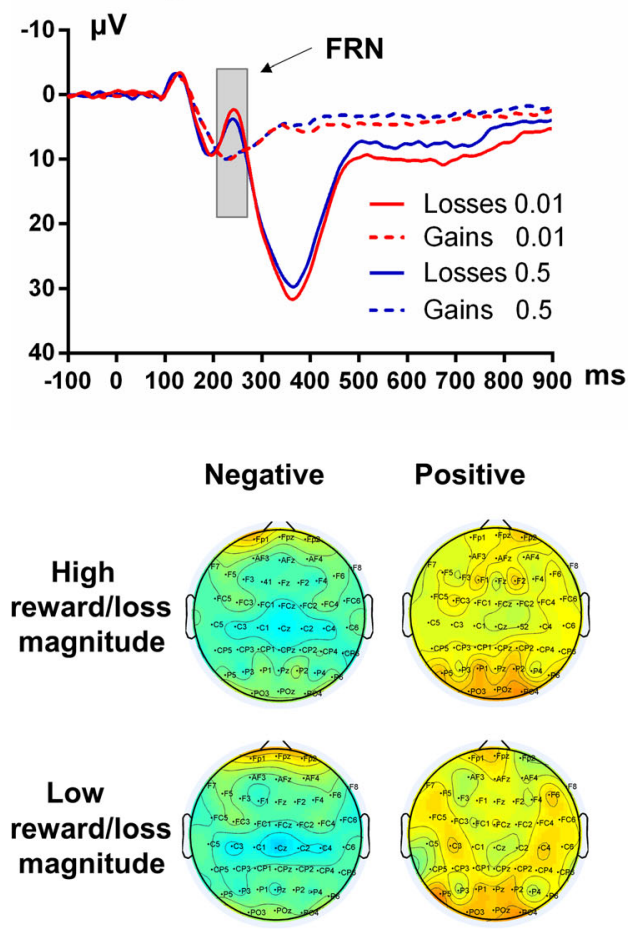

Fig. 2. Grand-averaged ERP waveforms for gains and losses according to reward/loss magnitude condition and sensation-seeking group at $\mathrm{Cz}$. The time window of FRN for low and high reward/loss magnitude

behavior) between high and low magnitude conditions after negative feedback in both sensation-seeking groups because previous studies have consistently suggested that FRN response might predict future decision-making behavior (Ridderinkhof et al., 2004; Xu et al., 2018).

\section{Results}

\section{Demographic characteristics and behavioral results}

A summary of the demographic characteristics of participants is reported in Table 1 for both the LSS and HSS groups. We observed no differences in age, education level, or gender between the sensation-seeking groups (all $p>0.050$ ), while the sensation-seeking score and subdimension scores were

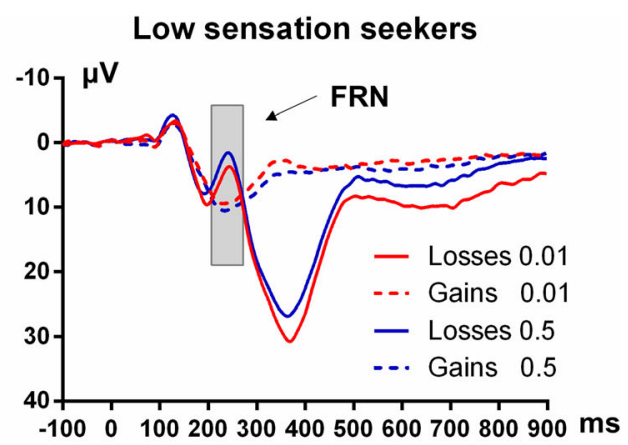

$15 \mu \mathrm{V}$
$0 \mu \mathrm{V}$
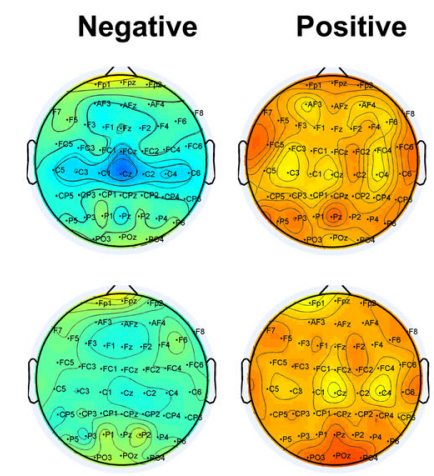

conditions is marked with the shaded areas. Scalp maps (200-280 ms) show the topography for the FRN by condition and outcome likelihood

significantly different between LSS and HSS groups (all $p<$ $0.001)$.

We examined the effects of reward/loss magnitude as a function of sensation-seeking group on the average number of pumps following positive (reward) and negative (loss) feedback. A 2 (group: HSS vs. LSS) $\times 2$ (reward/loss magnitude: 0.01 vs. 0.5$) \times 2$ (feedback valence: positive vs. negative) mixed ANOVA was conducted, with the average number of pumps following positive or negative feedback as the dependent variable. We found a significant three-way interaction between group, reward/loss magnitude, and feedback $(F(1$, $\left.40)=5.30, p=0.027, \eta_{\mathrm{p}}{ }^{2}=0.117\right)$, and a significant main effect of feedback $\left(F(1,40)=37.28, p<0.001, \eta_{\mathrm{p}}{ }^{2}=0.482\right)$. Participants made fewer pumps following negative (loss) feedback than following positive (reward) feedback.

We then separately analyzed the average number of pumps following positive and negative feedbacks using a 2 (group:

Table 2 Number of accepted trials in each condition $(\mathrm{M} \pm \mathrm{SD})$

\begin{tabular}{|c|c|c|c|c|c|c|c|c|}
\hline \multirow[t]{3}{*}{ Feedback } & \multicolumn{4}{|l|}{ HSS } & \multicolumn{4}{|l|}{ LSS } \\
\hline & \multicolumn{2}{|c|}{ Low magnitude } & \multicolumn{2}{|c|}{ High magnitude } & \multicolumn{2}{|c|}{ Low magnitude } & \multicolumn{2}{|c|}{ High magnitude } \\
\hline & $M$ & $S D$ & $M$ & $S D$ & $M$ & $S D$ & $M$ & $S D$ \\
\hline Positive & 36.85 & 9.84 & 38.64 & 9.72 & 36.02 & 9.51 & 37.94 & 9.68 \\
\hline Negative & 37.23 & 8.68 & 39.28 & 8.48 & 37.83 & 8.57 & 37.08 & 8.76 \\
\hline
\end{tabular}


HSS vs. LSS $) \times 2($ reward/loss magnitude: 0.01 vs. 0.5$)$ ANOVA, respectively. Following positive feedback, we observed no significant main effects of group $(F(1,40)=0.43, p$ $=0.514)$ or reward magnitude/loss $(F(1,40)=0.08, p=$ 0.782 ), and no significant interaction between group and reward magnitude/loss $(F(1,40)=3.11, p=0.085)$. In contrast, following negative feedback, we observed a significant interaction between group and reward/loss magnitude $(F(1,40)=$ $\left.10.14, p=0.003, \eta_{\mathrm{p}}{ }^{2}=0.202\right)$, but no main effect of group $(F(1,40)=1.20, p=0.280)$ or reward/loss magnitude $(F(1$, $40)=0.26, p=0.611$, Table 3 ). Further post-hoc comparisons indicated that the average number of pumps was lower in the high magnitude reward/loss condition $(5.23 \pm 0.88)$ than in the low $(5.97 \pm 1.25)$ magnitude reward/loss condition for the LSS individuals $\left(F(1,20)=12.31, p=0.002, \eta_{\mathrm{p}}{ }^{2}=0.381\right)$, whereas the average number of pumps was comparable between the high $(6.18 \pm 1.22)$ and low $(5.64 \pm 1.02)$ magnitude conditions for the HSS individuals $(F(1,20)=3.91, p=$ 0.062 ).

\section{ERP results}

Figure 2 shows the grand-average ERP waveforms at the $\mathrm{Cz}$ site. A 2 (group: HSS vs. LSS) $\times 2$ (reward/loss magnitude condition: 0.01 vs. 0.5$) \times 2$ (feedback valence: positive vs. negative) mixed ANOVA was conducted with FRN (defined as the mean amplitude of the wave in the 200-280 ms time window after feedback) as the dependent variable. We observed a main effect of feedback valence $(F(1,40)=16.97$, $\left.p<0.001, \eta_{\mathrm{p}}^{2}=0.298\right)$, with ERP responses being more negative after negative feedback $(4.80 \mu \mathrm{V})$ than those after positive feedback $(8.88 \mu \mathrm{V})$. We also observed a significant group $\times$ reward/loss magnitude $\times$ feedback valence interaction $\left(F(1,40)=8.07, p=0.007, \eta_{\mathrm{p}}{ }^{2}=0.168\right.$; Table 3 ; Supplementary Figure 1).

When only examining ERP responses to positive feedback, we did not observe a significant group $\times$ reward/loss magnitude interaction $(F(1,40)=0.48, p=0.492)$, whereas there was such an interaction for responses to negative feedback
$\left(F(1,40)=9.62, p=0.004, \eta_{\mathrm{p}}{ }^{2}=0.194\right)$. Further post-hoc comparisons indicated that ERP responses were more negative in the high magnitude condition $(3.49 \mu \mathrm{V})$ than in the low magnitude condition $(5.50 \mu \mathrm{V})$ for the LSS individuals $(F(1$, $\left.20)=11.49, p=0.002, \eta_{\mathrm{p}}^{2}=0.223\right)$. However, no such differences were found for ERP responses to negative feedback between the high $(5.41 \mu \mathrm{V})$ and low $(4.82 \mu \mathrm{V})$ magnitude conditions for the HSS individuals $(F(1,20)=0.99, p=$ $0.325)$.

We also performed correlation analysis to examine whether differences in FRN correlated with differences in uncertaintybearing behavior between the small and large reward/loss magnitude conditions in the whole sample as well as in the LSS or HSS groups separately. We found a significant positive correlation between ?FRN and ?decision-making behavior under uncertainty after negative feedback for the whole sample $(r=0.49, p=0.002)$. Similar correlation was found in the LSS group $(r=0.48, p=0.027)$, but not in the HSS group $(r=0.26$, $p=0.254)$, and the difference between correlations for both groups did not reach significance $(Z=0.78, p=0.441)$.

\section{Discussion}

In the present study, we used event-related potential (ERP) with the BART paradigm and investigated how individuals' sensation seeking modulates their brain responses to sequential decision-making with variable reward/loss outcome magnitudes. We found that sensation seeking significantly modulated the effect of reward/loss magnitude variations on decision-making under uncertainty, with LSS being more uncertainty-averse in the high magnitude reward/loss condition compared with the low magnitude condition, whereas HSS showed no change between high and low rewards/loss magnitude conditions. More importantly, this difference in sequential uncertainty-bearing behavior between sensation seekers was consistently reflected by their EEG signals in the outcome appraisal stage, as indexed by the FRN. Combined with the correlations between ?decision-making

Table 3 Average number of pumps and mean amplitude of FRN as a function of feedback and reward/loss magnitude (M $\pm \mathrm{SD})$

\begin{tabular}{|c|c|c|c|c|c|c|c|c|}
\hline \multirow{3}{*}{$\begin{array}{l}\text { Feedback } \\
\text { AP }\end{array}$} & \multicolumn{4}{|l|}{ HSS } & \multicolumn{4}{|l|}{ LSS } \\
\hline & \multicolumn{2}{|c|}{ Low magnitude } & \multicolumn{2}{|c|}{ High magnitude } & \multicolumn{2}{|c|}{ Low magnitude } & \multicolumn{2}{|c|}{ High magnitude } \\
\hline & $M$ & $S D$ & $M$ & $S D$ & $M$ & $S D$ & $M$ & $S D$ \\
\hline Positive & 6.23 & 0.99 & 6.52 & 1.20 & 6.39 & 1.10 & 5.99 & 1.04 \\
\hline Negative & 5.64 & 1.02 & 6.18 & 1.22 & 5.97 & 1.25 & 5.23 & 0.88 \\
\hline \multicolumn{9}{|l|}{ FRN $(\mu \mathrm{V})$} \\
\hline Positive & 8.41 & 3.53 & 8.53 & 3.39 & 9.00 & 3.66 & 9.56 & 3.57 \\
\hline Negative & 4.82 & 6.90 & 5.41 & 5.92 & 5.50 & 6.24 & 3.49 & 5.59 \\
\hline
\end{tabular}

$\mathrm{AP}=$ Average number of pumps following positive or negative feedback 
behavior under uncertainty and ?FRN following negative feedback in the BART, these findings provide insights into the neural mechanisms underlying sequential uncertaintybearing behavior in sensation seeking and suggest that FRN response might be a biological marker to predict future decision-making behavior following negative feedback.

Our behavioral results showed that sensation seeking moderated the effect of reward/loss magnitude on uncertaintybearing behavior in the BART. These findings suggest that compared to LSS individuals, HSS individuals are hyposensitive to potential losses. The current findings are in line with previous studies employing various decision-making tasks, including the BART (Bornovalova et al., 2009; Xu et al., 2013), Iowa Gambling Task (Penolazzi, Leone, \& Russo, 2013), and the probability discounting task (Zheng, Tian, Li, $\&$ Liu, 2019). However, participants in the previous studies were instructed to make decisions in a losses and/or gains context, which suggested that a hypoactive avoidance (punishment) system or a hyperactive approach (reward) system may regulate risk decision-making behavior in sensation seeking. The current study focuses on the uncertainty-bearing behavior following positive or negative feedback in a dynamic decision-making context and strengthens that heightened uncertainty-bearing behavior in sensation seeking may be likely driven by a hypoactive avoidance system rather than a hyperactive approach system.

The FRN amplitude in the frontocentral region was modulated by the feedback valence, and the negative feedback induced a larger response than the positive feedback. Furthermore, we found significantly greater FRN amplitudes in response to high-magnitude negative feedback compared with low-magnitude negative feedback, indicating that reward/loss magnitude modulates in-brain activity related to decision-making under uncertainty. Researchers have obtained diverging evidence concerning the sensitivity of the FRN to the amount lost (Goyer, Woldorff, \& Huettel, 2008). One interpretation for these findings is that FRN is sensitive to reinforcement learning and the motivational significance of the outcome (Gehring \& Willoughby, 2002; Nieuwenhuis et al., 2004). The high magnitude reward/loss condition might have enhanced the expectancy of the outcome, therefore changing participants' desire for gain and avoidance of loss, leading to greater prediction errors.

We also found that neural responses to decision-making under uncertainty are modulated by sensation seeking. Sensitivity to uncertainty in the FRN only appeared for LSS individuals - that is, sensitivity to uncertainty was greater in the high magnitude reward/loss condition relative to the low magnitude condition in LSS individuals but was comparable between conditions among HSS individuals. This finding is consistent with the prediction of the optimal arousal theory (Zuckerman, 1994), which holds that HSS individuals are chronically under-aroused and require more intense or novel stimulation to reach their optimal level of arousal. Thus, stimulation (our high vs. low reward/loss magnitude parameters) that is suitable for LSS individuals might fail to arouse HSS individuals (Zheng \& Liu, 2015). Our findings also provide some support for motivational theory (Joseph et al., 2009; Lissek et al., 2005) as the blunted FRN to negative outcomes for different reward magnitudes in HSS (compared with LSS) is consistent with the hypoactive avoidance system in sensation seeking. Previous research also has found that sensation seeking is associated with a deficient brain response to monetary punishment (Kruschwitz et al., 2012) and a reduced negative bias (Zheng et al., 2011).

According to principles of reinforcement learning, the FRN reflects neural activity in the anterior cingulate cortex (Holroyd \& Coles, 2002), a region that plays a key role in feedback evaluation and learning about the consequences of actions to select more appropriate future behaviors (Ridderinkhof et al., 2004). The FRN not only reflects whether the current feedback is good or bad but also it is correlated with future decision-making behavior. In line with this view, significant correlations were found between ?decision-making behavior (changes in the average number of pumps between high and low magnitude conditions) and ?FRN (changes at higher magnitude versus lower magnitude) following negative feedback in the BART. These findings align with the findings from numerous previous studies showing that FRN was more negatively oriented following monetary losses when participants chose a different option on the subsequent trial (Holroyd \& Coles, 2008; Holroyd \& Krigolson, 2007; Meyer, Bekkering, Janssen, de Bruijn, \& Hunnius, 2014; van der Helden, Boksem, \& Blom, 2009).

We failed to find any support for the hyperactive approach system in sensation seeking, according to which HSS individuals should demonstrate enhanced activation after receiving monetary rewards or other positive stimuli (Joseph et al., 2009; Kruschwitz et al., 2012). A possible explanation for this null finding may be the link between high-level sensationseeking and the dysfunctional dopaminergic system (Comings \& Blum, 2000), which leads to a more sluggish motivational system (Derringer et al., 2010). Consistent with this idea, previous studies have shown that the noveltyseeking personality traits were associated with reduced dopamine D2/D3 receptor availability in the dopaminergic brain regions, including the substantia nigra, ventral tegmental area, insular cortex, ventral striatum, and globus pallidus (Caravaggio et al., 2016; Zald et al., 2008). These results suggest a specific inverse relationship between the noveltyseeking trait and auto receptor availability in the dopaminergic systems. These findings are inconsistent with findings from a previous study (Schmidt, Holroyd, Debener, \& Hwig, 2017). In that study, participants were instructed to make decisions in a gain context, in which they choose one of the four cards and won either a small ( 1 cent) or large (10 cents) reward that was 
given to them either at the end of the experiment or after 6 months. The larger FRN/reward positivity (RewP) amplitude, when calculated as the difference between delayed reward and immediate gains, was associated with high impulsivity and less self-control (Schmidt et al., 2017). In contrast, in our study, participants were instructed to make decisions in a context of combined gains and losses, in which stopping balloon inflation and conserving earning is a safe option with outcomes that participants expected, therefore the FRN are not informative. This is in line with the reinforcement-learning theory by Holroyd and Coles (2002), which predicts larger FRN amplitudes following unexpected outcomes than those following expected outcomes. It also is plausible that the larger FRN/reward positivity amplitude may be linked to high impulsivity, because large reward positivity amplitude to monetary rewards has been reported for high reward discounting (Cherniawsky \& Holroyd, 2013), and high-risk individuals may discount delayed rewards more sharply and score higher on the self-report impulsivity measure relative to the low-risk participants (Crean, de Wit, \& Richards, 2000).

Our findings should nevertheless be considered in light of several limitations. The primary limitation concerns the modest sample size of $21 \mathrm{HSS}$ and 21 LSS participants as well as the fact that the sample came from a homogenous population of college students. These sample characteristics limit our ability to generalize our findings to more diverse and clinically impaired groups. At the same time, most existing sensationseeking studies have recruited college students as participants; thus, this limitation applies not only to our study but to the literature in general. Additionally, we adopted the criteria of a previous study (Xu et al., 2016) and excluded participants who reported having drunk alcohol or smoked tobacco within 2 weeks of the test. However, we still cannot completely rule out the possible impact of smoking or drinking on current outcomes, given that many studies demonstrated that alcohol or tobacco dependence is associated with poor decisionmaking under uncertainty (Brevers et al., 2014; Cavalca et al., 2013; Luhar et al., 2013). Therefore, more representative samples are required in future studies to generalize these findings to more populations of interest. Another limitation relates to the experimental task. Although we used different amounts of money for the low and high magnitude conditions ( 1 cent vs. 50 cents per pump) in the BART task, even the high magnitude rewards (50 cents per pump) were still quite a low amount of money in the real world. However, we still found differential modulation effects of reward/loss magnitude variations on the BART behavior and the FRN between LSS and HSS individuals. Replication studies should adopt much larger monetary rewards to validate these findings. The third limitation involves the measurement of sensation seeking we used. In this study, we employed the SSS-V, which focuses only on action and pays no attention to potential harm (Zuckerman, 1994). Moreover, it does not consider other dimensions of risk avoidance or approach (e.g., other aspects of behavioral impulsivity such as behavioral inhibition or delay discounting). Therefore, future studies are necessary to validate the current findings using a more sophisticated sensation seeking measurement.

\section{Conclusions}

We observed behavioral differences in sensitivity to uncertainty between HSS and LSS individuals during a sequential decision-making task, which is consistent with the findings of previous studies (Bornovalova et al., 2009; Xu, et al., 2013). More importantly, we also found differences in neural responses to negative feedback on the BART between these groups. HSS individuals exhibited no differences in the FRN between different reward magnitude conditions, whereas LSS individuals did. These findings suggest that a high level of sensation seeking is associated with behavioral and neural insensitivity to negative outcomes during decision-making, which might lead to more risky behavior in these individuals when facing potential losses.

Acknowledgements This research was supported in part by the National Natural Science Foundation of China (31400872), the Ministry of Education of Humanities and Social Science Project (18YJA630123), and the Shanghai Pujiang Program (17PJC090).

Contributors Conceived and designed the experiments: XS, RH; Performed the experiment: XS, WH, WC, XZ; Analyzed the data: XS; Wrote the paper: XS, LL, ZK, RH.

\section{Compliance with ethical standards}

Conflict of interest statement The authors declare that the research was conducted in the absence of any commercial or financial relationships that could be construed as a potential conflict of interest.

\section{References}

Banducci, A. N., Felton, J. W., Dahne, J., Ninnemann, A., \& Lejuez, C. (2015). Maternal risk taking on the balloon analogue risk task as a prospective predictor of youth alcohol use escalation. Addictive behaviors, 49, 40-45. https://doi.org/10.1016/j.addbeh.2015.05.011

Bardo, M. T., Williams, Y., Dwoskin, L. P., Moynahan, S., Perry, I., \& Martin, C. A. (2007). The sensation seeking trait and substance use: Research findings and clinical implications. Current Psychiatry Reviews, 3(1), 3-13. https://doi.org/10.2174/157340007779815682

Bellebaum, C., Polezzi, D., \& Daum, I. (2010). It is less than you expected: the feedback-related negativity reflects violations of reward magnitude expectations. Neuropsychologia, 48(11), 3343-3350. https://doi.org/10.1016/j.neuropsychologia.2010

Brevers, D., Bechara, A., Cleeremans, A., Kornreich, C., Verbanck, P., \& Noël, X. (2014). Impaired Decision-Making Under Risk in Individuals with Alcohol Dependence. Alcoholism: Clinical and Experimental Research, 38(7), 1924-1931. https://doi.org/10.1111/ acer. 12447 
Boorman, E. D., Behrens, T. E. J., Woolrich, M. W., \& Rushworth, M. F. S. (2009). How Green Is the Grass on the Other Side? Frontopolar Cortex and the Evidence in Favor of Alternative Courses of Action. Neuron, 62(5), 733-743. https://doi.org/10. 1016/j.neuron.2009.05.014

Bornovalova, M. A., Cashman-Rolls, A., O'donnell, J. M., Ettinger, K., Richards, J. B., \& Lejuez, C. (2009). Risk taking differences on a behavioral task as a function of potential reward/loss magnitude and individual differences in impulsivity and sensation seeking. Pharmacology Biochemistry and Behavior, 93(3), 258-262. https:// doi.org/10.1016/j.pbb.2008.10.023

Caravaggio, F., Fervaha, G., Chung, J. K., Gerretsen, P., Nakajima, S., Plitman, E., ... Graff-Guerrero, A. (2016). Exploring personality traits related to dopamine D2/3 receptor availability in striatal subregions of humans. European Neuropsychopharmacol, 26(4), 644652. https://doi.org/10.1016/j.euroneuro.2016.02.010

Cavalca, E., Kong, G., Liss, T., Reynolds, E. K., Schepis, T. S., Lejuez, C. W., \& Krishnan-Sarin, S. (2013). A preliminary experimental investigation of peer influence on risk-taking among adolescent smokers and non-smokers. Drug and Alcohol Dependence, 129(1-2), 163166. https://doi.org/10.1016/j.drugalcdep.2012.09.020

Cherniawsky, A. S., \& Holroyd, C. B. (2013). High temporal discounters overvalue immediate rewards rather than undervalue future rewards: an event-related brain potential study. Cognitive, Affective \& Behavioral Neuroscience, 13(1), 36-45. https://doi.org/10.3758/ s13415-012-0122-x

Comings, D. E., \& Blum, K. (2000). Reward deficiency syndrome: genetic aspects of behavioral disorders. Progress in Brain Research, 126, 325-341. https://doi.org/10.1016/s0079-6123(00)26022-6

Crean, J. P., de Wit, H., \& Richards, J. B. (2000). Reward discounting as a measure of impulsive behavior in a psychiatric outpatient population. Experimental and Clinical Psychopharmacology, 8(2), 155162. https://doi.org/10.1037/1064-1297.8.2.155

Cservenka, A., Herting, M. M., Seghete, K. L. M., Hudson, K. A., \& Nagel, B. J. (2013). High and low sensation seeking adolescents show distinct patterns of brain activity during reward processing. NeuroImage, 66, 184-193. https://doi.org/10.1016/j.neuroimage. 2012.11.003

Delorme, A., \& Makeig, S. (2004). EEGLAB: an open source toolbox for analysis of single-trial EEG dynamics including independent component analysis. Journal of neuroscience methods, 134(1), 9-21. https://doi.org/10.1016/j.jneumeth.2003.10.009

Derringer, J., Krueger, R. F., Dick, D. M., Saccone, S., Grucza, R. A., Agrawal, A., ... Gene Environment Association Studies, C (2010). Predicting sensation seeking from dopamine genes. A candidatesystem approach. Psycholological Science, 21(9), 1282-1290. https://doi.org/10.1177/0956797610380699

Doran, N., Sanders, P. E., Bekman, N. M., Worley, M. J., Monreal, T. K., McGee, E., ... Brown, S. A. (2011). Mediating influences of negative affect and risk perception on the relationship between sensation seeking and adolescent cigarette smoking. Nicotine \& Tobacco Research, ntr025. https://doi.org/10.1093/ntr/ntr025

Euser, A. S., Van Meel, C. S., Snelleman, M., \& Franken, I. H. (2011). Acute effects of alcohol on feedback processing and outcome evaluation during risky decision-making: an ERP study. Psychopharmacology, 217(1), 111. https://doi.org/10.1007/s00213011-2264

Fein, G., \& Chang, M. (2008). Smaller feedback ERN amplitudes during the BART are associated with a greater family history density of alcohol problems in treatment-naive alcoholics. Drug and alcohol dependence, 92(1), 141-148. https://doi.org/10.1016/j.drugalcdep. 2007.07.017

Fillmore, M. T., Ostling, E. W., Martin, C. A., \& Kelly, T. H. (2009). Acute effects of alcohol on inhibitory control and information processing in high and low sensation-seekers. Drug and Alcohol
Dependence, 100(1-2), 91-99. https://doi.org/10.1016/j.drugalcdep. 2008.09.007

Gehring, W. J., \& Willoughby, A. R. (2002). The medial frontal cortex and the rapid processing of monetary gains and losses. Science, 295(5563), 2279-2282. https://doi.org/10.1126/science.1066893

Goyer, J. P., Woldorff, M. G., \& Huettel, S. A. (2008). Rapid electrophysiological brain responses are influenced by both valence and magnitude of monetary rewards. Journal of Cognitive Neuroscience, 20(11), 2058-2069. https://doi.org/10.1162/jocn.2008.20134

Gu, R., Jiang, Y., Kiser, S., Black, C. L., Broster, L. S., Luo, Y., \& Kelly, T. H. (2017). Impulsive personality dimensions are associated with altered behavioral performance and neural responses in the monetary incentive delay task. Neuropsychologia, 103, 59-68. https://doi. org/10.1016/j.neuropsychologia.2017.07.013

Gu, R., Lei, Z., Broster, L., Wu, T., Jiang, Y., \& Luo, Y.-J. (2011). Beyond valence and magnitude: A flexible evaluative coding system in the brain. Neuropsychologia, 49(14), 3891-3897. https://doi.org/10. 1016/j.neuropsychologia.2011

Harris, N., Newby, J., \& Klein, R. G. (2015). Competitiveness facets and sensation seeking as predictors of problem gambling among a sample of university student gamblers. Journal of Gambling Studies, 31(2), 385-396. https://doi.org/10.1007/s10899-013-9431-4

Hebb, D. O. (1955). Drives and the CNS (conceptual nervous system). Psychological review, 62(4), 243. https://doi.org/10.1037/h0041823

Helfinstein, S. M., Schonberg, T., Congdon, E., Karlsgodt, K. H., Mumford, J. A., Sabb, F. W., ... Poldrack, R. A. (2014). Predicting risky choices from brain activity patterns. Proceedings of the National Academy of Sciences, 111(7), 2470-2475. https://doi. org/10.1073/pnas.1321728111

Holroyd, C. B., \& Coles, M. G. (2002). The neural basis of human error processing: reinforcement learning, dopamine, and the error-related negativity. Psychological review, 109(4), 679. https://doi.org/10. 1037/0033-295x.109.4.679

Holroyd, C. B., \& Coles, M. G. (2008). Dorsal anterior cingulate cortex integrates reinforcement history to guide voluntary behavior. cortex, 44(5), 548-559. https://doi.org/10.1016/j.cortex.2007.08.013

Holroyd, C. B., Hajcak, G., \& Larsen, J. T. (2006). The good, the bad and the neutral: electrophysiological responses to feedback stimuli. Brain research, 1105(1), 93-101. https://doi.org/10.1016/j.brainres. 2005.12.015

Holroyd, C. B., \& Krigolson, O. E. (2007). Reward prediction error signals associated with a modified time estimation task. Psychophysiology, 44(6), 913-917. https://doi.org/10.1111/j.14698986.2007.00561.x

Horvath, P., \& Zuckerman, M. (1993). Sensation seeking, risk appraisal, and risky behavior. Personality and Individual Differences, 14(1), 41-52. https://doi.org/10.1016/0191-8869(93)90173-Z

Iversen, H., \& Rundmo, T. (2002). Personality, risky driving and accident involvement among Norwegian drivers. Personality \& Individual Differences, 33, 1251-1263. https://doi.org/10.1016/S01918869(02)00010-7

Jonah, B. A. (1997). Sensation seeking and risky driving: a review and synthesis of the literature. Accident Analysis and Prevention, 29(5), 651-665. https://doi.org/10.1016/s0001-4575(97)00017-1

Joseph, J. E., Liu, X., Jiang, Y., Lynam, D., \& Kelly, T. H. (2009). Neural correlates of emotional reactivity in sensation seeking. Psychological Science, 20(2), 215-223. https://doi.org/10.1111/j. 1467-9280.2009.02283.x

Kóbor, A., Takács, Á., Janacsek, K., Németh, D., Honbolygó, F., \& Csépe, V. (2015). Different strategies underlying uncertain decision making: Higher executive performance is associated with enhanced feedback-related negativity. Psychophysiology, 52(3), 367-377. https://doi.org/10.1111/psyp.12331

Kessler, L., Hewig, J., Weichold, K., Silbereisen, R. K., \& Miltner, W. H. (2017). Feedback negativity and decision-making behavior in the Balloon Analogue Risk Task (BART) in adolescents is modulated 
by peer presence. Psychophysiology, 54(2), 260-269. https://doi.org/ 10.1111/psyp. 12783

Kiat, J., Straley, E., \& Cheadle, J. E. (2015). Escalating risk and the moderating effect of resistance to peer influence on the P200 and feedback-related negativity. Social cognitive and affective neuroscience, 11(3), 377-386. https://doi.org/10.1093/scan/nsv121

Kruschwitz, J. D., Simmons, A. N., Flagan, T., \& Paulus, M. P. (2012). Nothing to lose: processing blindness to potential losses drives thrill and adventure seekers. Neuroimage, 59(3), 2850-2859. https://doi. org/10.1016/j.neuroimage.2011.09.048

Lang, K., Murphy, J. G., Monahan, C. J., Dennhardt, A. A., Skidmore, J. R., \& McDevitt-Murphy, M. E. (2012). The role of positive consequences of alcohol in the relation between sensation seeking and drinking. Addiction Research \& Theory, 20(6), 504-510. https:// doi.org/10.3109/16066359.2012.667854

Leicht, G., Troschütz, S., Andreou, C., Karamatskos, E., Ertl, M., Naber, D., \& Mulert, C. (2013). Relationship between oscillatory neuronal activity during reward processing and trait impulsivity and sensation seeking. PloS one, 8(12), e83414. https://doi.org/10.1371/journal. pone. 0083414

Lejuez, C. W., Read, J. P., Kahler, C. W., Richards, J. B., Ramsey, S. E., Stuart, G. L., ... Brown, R. A. (2002). Evaluation of a behavioral measure of risk taking: the Balloon Analogue Risk Task (BART). Journal of Experimental Psychology: Applied, 8(2), 75. https://doi. org/10.1037/1076-898x.8.2.75

Lissek, S., Baas, J. M., Pine, D. S., Orme, K., Dvir, S., Rosenberger, E., \& Grillon, C. (2005). Sensation seeking and the aversive motivational system. Emotion, 5(4), 396-407. https://doi.org/10.1037/1528-3542. 5.4.396

Luck, S. J. (2014). An introduction to the event-related potential technique. Cambridge, MA: MIT Press.

Luhar, R., Sawyer, K., Gravitz, Z., Mosher Ruiz, S., \& Oscar-Berman, M. (2013). Brain volumes and neuropsychological performance are related to current smoking and alcoholism history. Neuropsychiatric Disease and Treatment, 1767. https://doi.org/10.2147/NDT.S52298

Luo, Q., \& Qu, C. (2013). Comparison enhances size sensitivity: neural correlates of outcome magnitude processing. PloS one, 8(8), e71186. https://doi.org/10.1371/journal.pone.0071186

Marco-Pallares, J., Cucurell, D., Münte, T. F., Strien, N., \& RodriguezFornells, A. (2011). On the number of trials needed for a stable feedback-related negativity. Psychophysiology, 48(6), 852-860. https://doi.org/10.1111/j.1469-8986.2010.01152.x

Massar, S. A. A., Rossi, V., Schutter, D. J. L. G., \& Kenemans, J. L. (2012). Baseline EEG theta/beta ratio and punishment sensitivity as biomarkers for feedback-related negativity (FRN) and risk-taking. Clinical Neurophysiology, 123(10), 1958-1965. https://doi.org/ 10.1016/j.clinph.2012.03.005

Meyer, M., Bekkering, H., Janssen, D. J., de Bruijn, E. R., \& Hunnius, S. (2014). Neural correlates of feedback processing in toddlers. Journal of Cognitive Neuroscience, 26(7), 1519-1527. https://doi. org/10.1162/jocn_a_00560

Miltner, W. H. R., Braun, C. H., \& Coles, M. G. H. (1997). Event-Related Brain Potentials Following Incorrect Feedback in a Time-Estimation Task: Evidence for a "Generic" Neural System for Error Detection. Journal of Cognitive Neuroscience, 9(6), 788-798. https://doi.org/ 10.1162/jocn.1997.9.6.788

Mishra, S., Lalumière, M. L., \& Williams, R. J. (2010). Gambling as a form of risk-taking: Individual differences in personality, riskaccepting attitudes, and behavioral preferences for risk. Personality and Individual Differences, 49(6), 616-621. https:// doi.org/10.1016/j.paid.2010.05.032

Nieuwenhuis, S., Yeung, N., Holroyd, C. B., Schurger, A., \& Cohen, J. D. (2004). Sensitivity of electrophysiological activity from medial frontal cortex to utilitarian and performance feedback. Cerebral Cortex, 14(7), 741-747. https://doi.org/10.1093/cercor/bhh034
Novak, B. K., Novak, K. D., Lynam, D. R., \& Foti, D. (2016). Individual differences in the time course of reward processing: Stage-specific links with depression and impulsivity. Biological Psychology, 119, 79-90. https://doi.org/10.1016/j.biopsycho.2016.07.008

Penolazzi, B., Leone, L., \& Russo, P. M. (2013). Individual Differences and Decision Making: When the Lure Effect of Gain Is a Matter of Size. PLoS ONE, 8(3), e58946. https://doi.org/10.1371/journal. pone. 0058946

Ridderinkhof, K. R., Ullsperger, M., Crone, E. A., \& Nieuwenhuis, S. (2004). The role of the medial frontal cortex in cognitive control. Science, 306(5695), 443-447. https://doi.org/10.1126/science. 1100301

Ruedl, G., Burtscher, M., Wolf, M., Ledochowski, L., Bauer, R., Benedetto, K. P., \& Kopp, M. (2015). Are self-reported risk-taking behavior and helmet use associated with injury causes among skiers and snowboarders? Scandinavian Journal of Medcine and Science in Sports, 25(1), 125-130. https://doi.org/10.1111/sms.12139

Schmidt, B., \& Hewig, J. (2015). Paying Out One or All Trials: A Behavioral Economic Evaluation of Payment Methods in a Prototypical Risky Decision Study. The Psychological Record, 65(2), 245-250. https://doi.org/10.1007/s40732-014-0112-1

Schmidt, B., Holroyd, C. B., Debener, S., \& Hewig, J. (2017). I can't wait! Neural reward signals in impulsive individuals exaggerate the difference between immediate and future rewards: Brain responses to waiting. Psychophysiology, 54(3), 409-415. https://doi.org/10. 1111/psyp. 12796

Schmidt, B., Keßler, L., Hecht, H., Hewig, J., Holroyd, C. B., \& Miltner, W. H. R. (2019). What you give is what you get: Payment of one randomly selected trial induces risk-aversion and decreases brain responses to monetary feedback. Cognitive, Affective, \& Behavioral Neuroscience, 19(1), 187-196. https://doi.org/10.3758/ s13415-018-00656-1

Schmidt, B., Mussel, P., \& Hewig, J. (2013). I'm too calm-Let's take a risk! On the impact of state and trait arousal on risk taking: I'm too calm-Let's take a risk! Psychophysiology, 50(5), 498-503. https:// doi.org/10.1111/psyp.12032

Schonberg, T., Fox, C. R., Mumford, J. A., Congdon, E., Trepel, C., \& Poldrack, R. A. (2012). Decreasing ventromedial prefrontal cortex activity during sequential risk-taking: an fMRI investigation of the balloon analog risk task. Frontiers in neuroscience, 6, 80. https:// doi.org/10.3389/fnins.2012.00080

van der Helden, J., Boksem, M. A., \& Blom, J. H. (2009). The importance of failure: feedback-related negativity predicts motor learning efficiency. Cerebral Cortex, 20(7), 1596-1603. https://doi.org/10.1093/ cercor/bhp224

Wang, W., Wu, Y.-X., Peng, Z.-G., Lu, S.-W., Yu, L., Wang, G.-P., ... Wang, Y.-H. (2000). Test of sensation seeking in a Chinese sample. Personality and individual differences, 28(1), 169-179. https://doi. org/10.1016/s0191-8869(99)00092-6

Weiland, B. J., Welsh, R. C., Yau, W.-Y. W., Zucker, R. A., Zubieta, J.-K., \& Heitzeg, M. M. (2013). Accumbens functional connectivity during reward mediates sensation-seeking and alcohol use in high-risk youth. Drug and Alcohol Dependence, 128(1), 130-139. https://doi. org/10.1016/j.drugalcdep.2012.08.019

Wilson, L. C., \& Scarpa, A. (2011). The link between sensation seeking and aggression: a meta-analytic review. Aggressive Behavior, 37(1), 81-90. https://doi.org/10.1002/ab.20369

Wu, Y., \& Zhou, X. (2009). The P300 and reward valence, magnitude, and expectancy in outcome evaluation. Brain research, 1286, 114122. https://doi.org/10.1016/j.brainres.2009.06.032

Xu, S., Fang, Z., \& Rao, H. (2013). Real or hypothetical monetary rewards modulates risk taking behavior. Acta Psychologica Sinica, 45(8), 874-886. https://doi.org/10.3724/SP.J.1041.2013.00874

Xu, S., Pan, Y., Qu, Z., Fang, Z., Yang, Z., Yang, F., ... Rao, H. (2018). Differential effects of real versus hypothetical monetary reward 
magnitude on risk-taking behavior and brain activity. Scientific Reports, 8(1), 3712. https://doi.org/10.1038/s41598-018-21820-0

Xu, S., Pan, Y., Wang, Y., Spaeth, A. M., Qu, Z., \& Rao, H. (2016). Real and hypothetical monetary rewards modulate risk taking in the brain. Scientific Reports, 6. https://doi.org/10.1038/srep29520

Yeung, N., \& Sanfey, A. G. (2004). Independent coding of reward magnitude and valence in the human brain. Journal of Neuroscience, 24(28), 6258-6264. https://doi.org/10.1523/jneurosci.4537-03.2004

Zald, D. H., Cowan, R. L., Riccardi, P., Baldwin, R. M., Ansari, M. S., Li, R., ... Kessler, R. M. (2008). Midbrain dopamine receptor availability is inversely associated with novelty-seeking traits in humans. The Journal of Neuroscience, 28(53), 14372-14378. https://doi.org/10. 1523/jneurosci.2423-08.2008

Zheng, Y., \& Liu, X. (2015). Blunted neural responses to monetary risk in high sensation seekers. Neuropsychologia, 71, 173-180. https://doi. org/10.1016/j.neuropsychologia.2015.04.002.

Zheng, Y., Li, Q., Tian, M., Nan, W., Yang, G., Liang, J., \& Liu, X. (2017). Deficits in voluntary pursuit and inhibition of risk taking in sensation seeking: Risk Taking in Sensation Seeking. Human Brain Mapping, 38(12), 6019-6028. https://doi.org/10.1002/hbm. 23807

Zheng, Y., Sheng, W., Xu, J., \& Zhang, Y. (2014). Sensation seeking and error processing. Psychophysiology, 51(9), 824-833. https://doi.org/ $10.1111 /$ psyp. 12240
Zheng, Y., Tian, M., Li, Q., \& Liu, X. (2019). Greater tolerance to losses in sensation seeking: Evidence from probability and delay discounting. Drug and Alcohol Dependence, 194, 159-165. https://doi.org/10.1016/j.drugalcdep.2018.09.027

Zheng, Y., Xu, J., Jia, H., Tan, F., Chang, Y., Zhou, L., ... Qu, B. (2011). Electrophysiological correlates of emotional processing in sensation seeking. Biological Psychology, 88(1), 41-50. https://doi.org/10. 1016/j.biopsycho.2011.06.006.

Zhou, Z., Yu, R., \& Zhou, X. (2010). To do or not to do? Action enlarges the FRN and P300 effects in outcome evaluation. Neuropsychologia, 48(12), 3606-3613. https://doi.org/10.1016/j. neuropsychologia.2010.08.010

Zuckerman, M. (1994). Behavioral expressions and biosocial bases of sensation seeking: Cambridge University Press

Zuckerman, M., \& Kuhlman, D. M. (2000). Personality and risk-taking: common bisocial factors. Journal of personality, 68(6), 999-1029. https://doi.org/10.1111/1467-6494.00124

Publisher's note Springer Nature remains neutral with regard to jurisdictional claims in published maps and institutional affiliations. 